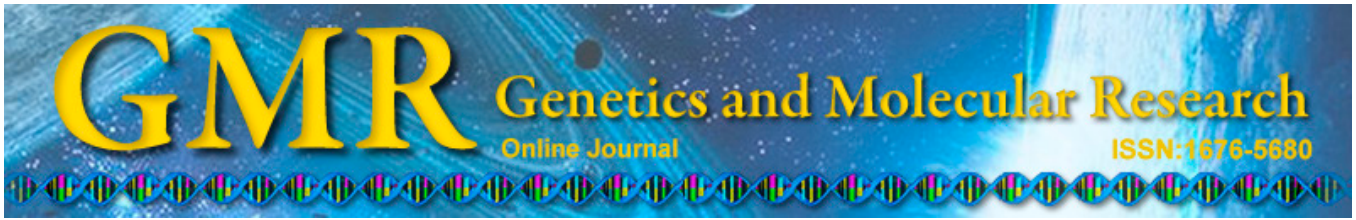

\title{
Correlation between the BRAF V600E mutation status and the clinicopathologic features of papillary thyroid carcinoma
}

\author{
C.L. Shi, Y. Sun, C. Ding, Y.C. Lv and H.D. Qin \\ The Fourth Department of General Surgery, \\ The Second Affiliated Hospital of Harbin Medical University, Harbin, China \\ Corresponding author: H.D. Qin \\ E-mail: huadongq2014@163.com
}

Genet. Mol. Res. 14 (3): 7377-7385 (2015)

Received August 12, 2014

Accepted November 27, 2014

Published July 3, 2015

DOI http://dx.doi.org/10.4238/2015.July.3.13

\begin{abstract}
This study sought to investigate the correlations of V-raf murine sarcoma viral oncogene homolog B1 (BRAF) gene mutations with the clinicopathologic features of papillary thyroid carcinoma and central lymph node metastasis. We retrospectively analyzed the 2-year medical records of patients who underwent surgery for papillary thyroid carcinoma. After screening, the records of 126 patients who met the study requirements were used to assess the characteristics associated with the BRAF V600E gene mutation. The BRAF mutation incidence rate among patients with papillary thyroid carcinoma was $69.0 \%$ (87/126). Univariate analysis revealed that the BRAF mutation status was correlated significantly with both tumor size and lymph node metastasis $(\mathrm{P}<0.05)$. Multivariate analysis revealed a significant correlation between lymph node metastasis and BRAF mutation status $(\mathrm{P}<0.05)$. When the tumor diameter was $\leq 10 \mathrm{~mm}$, the BRAF mutation status had no effect on lymph node metastasis $(\mathrm{P}>0.05)$. When the tumor diameter was $>10 \mathrm{~mm}$, the incidence of lymph node metastasis was significantly higher among BRAF mutation-positive patients
\end{abstract}


than among BRAF mutation-negative patients $(\mathrm{P}<0.05)$. BRAF gene mutations independently predicted central lymph node metastasis in patients with papillary thyroid carcinoma. For patients preoperatively diagnosed to be BRAF mutation-positive, the importance of central lymph node dissection should be emphasized because the tumor diameter increases; regional lymphatic and adipose tissue dissection should be routinely conducted. However, in mutation-negative patients with tumor diameters of $\leq 5 \mathrm{~mm}$, the need for central lymph node dissection should be re-examined.

Key words: Papillary thyroid carcinoma; BRAF; Gene mutation; Lymph node metastasis

\section{INTRODUCTION}

The biological behaviors of thyroid carcinoma vary, and cases can accordingly be classified as differentiated thyroid carcinomas, which are well differentiated and exhibit slow progression, or undifferentiated carcinomas, which are poorly differentiated and exhibit a poor prognosis (Montero et al., 2014). Papillary thyroid carcinoma (PTC) is the most common pathological type of thyroid carcinoma, accounting for $80-90 \%$ of all cases (Moo et al., 2010; Byrd et al., 2012). V-raf murine sarcoma viral oncogene homolog B1 (BRAF) mutations, which can induce tumor cell proliferation and differentiation (Kondo et al., 2006), represent the most common genetic event in thyroid carcinomas; this event has the highest incidence in PTC, occurring in 29-88\% of all cases (Kim et al., 2004; Mathur et al., 2011; Chakraborty et al., 2012). Currently, studies have identified more than 40 different BRAF gene mutations in human malignancies (Davies et al., 2002). The T1799A point mutation is the most common mutation, accounting for $90 \%$ of all BRAF gene mutations (Garnett and Marais, 2004).

The present study has proven that the BRAF V600E mutation is closely associated with the occurrence and development of PTC, and therefore, preoperative screening for BRAF gene mutations could help clinicians to develop therapeutic strategies that are more suitable for patients. However, the link between BRAF mutations and the specific clinicopathologic features of PTC remains controversial. Therefore, in this study, we analyzed the BRAF V600E mutation statuses of 126 patients with PTC and retrospectively analyzed their clinical records.

\section{MATERIAL AND METHODS}

\section{Subjects}

We retrospectively analyzed the clinical data of patients with unilateral PTC who were treated surgically. These patients had been admitted to the thyroid surgical department of our hospital between October 2011 and October 2013. After screening, the medical records of the 126 patients who met the study criteria were selected, and the corresponding pathological PTC tissue samples were collected. All subjects had been diagnosed by highly experienced pathologists, and difficult cases had been discussed and diagnosed by several pathologists. All patients underwent routine preoperative thyroid ultrasonography (GE Vivid 7 color Doppler ultrasonic diagnostic apparatus; probe frequency, $15 \mathrm{MHz}$; GE Healthcare, Milwaukee, 
WI, USA). All patients' preoperative levels of free triiodothyronine, free thyroxine, thyroidstimulating hormone (TSH), thyroglobulin antibodies, and thyroid peroxidase antibodies were detected via radioimmunoassay. Cases of Hashimoto's thyroiditis were confirmed on the basis of the presence of an elevated preoperative serum thyroid autoantibody level and postoperative pathology findings. All eligible patients met the following criteria: 1) no history of thyroid disease or the use of thyroid-related medications; 2) no history of Graves' ophthalmopathy and TSH receptor antibody positivity; 3 ) preoperative diagnosis of a unilateral thyroid mass with no suspicious lymph node enlargement in the neck area as determined by palpation and highfrequency ultrasonography; 4) PTC diagnostic confirmation via intraoperative rapid pathological and postoperative pathological assessments; and 5) treatment via total thyroid lobectomy on the affected side, isthmus resection, and central lymph node dissection on the affected side. Central neck lymph node dissection referred to the clearance of the lymphatic and adipose tissues located ventral to the trachea, near the trachea, and in the areas of the recurrent laryngeal nerve and prelaryngeal lymph nodes. The specific dissection area included all lymphatic and adipose tissues below the thyroid cartilage, above the sternal notch, and in the medial region of the carotid arteries (Sadowski et al., 2009). Clinicopathologic staging was conducted according to the seventh edition of the tumor-node-metastasis (TNM) staging system published by the American Joint Committee on Cancer in 2010 (Stratmann et al., 2012).

\section{Detection of the BRAF V600E mutation}

Genomic DNA isolated from the primary PTC was extracted from paraffin-embedded tissues. Sections with a confirmed tumor were deparaffinized and collected for DNA extraction. The process was performed using a spinal column procedure (AmoyDx ${ }^{\circledR}$ FFPE DNA Kit, Amoy Diagnostics, China) according to manufacturer instructions. The absorbance of the DNA samples was measured with a spectrophotometer, and the $\mathrm{A}_{260} / \mathrm{A}_{280}$ values were all between 1.8 and 2.0. The DNA samples were stored at $-20^{\circ} \mathrm{C}$ until real-time qualitative polymerase chain reaction analysis. The BRAF V600E mutation status of each primary PTC was determined using the AmoyDx BRAF V600E Mutation Detection Kit (Amoy Diagnostics). The mutant BRAF gene (encoding BRAF V600E) was amplified with specific primers and detected with novel probes using Bio-Rad CFX96 (Bio-Rad Laboratories, USA) according to manufacturer instructions. The FAM fluorescence signal was used to evaluate the mutation status of the sample. When the sample FAM Ct value was $\leq 28$, the sample was classified as negative or being below the detection limit of the kit. When the sample FAM Ct value was $<28$, the sample was classified as mutation-positive.

\section{Statistical analysis}

The statistical analysis was performed using the SPSS 13.0 statistical software package (SPSS Inc., Chicago, IL, USA). Measurement data are reported as means \pm standard deviations, and enumeration data are reported as frequencies and rates. According to the data type, the between-group comparisons were conducted using the independentsamples $t$-test (measurement) or $\chi^{2}$ test (enumeration). The effects of the clinicopathologic factors on the BRAF V600E mutation status and lymph node metastasis were evaluated using univariate and multivariate logistic regression analyses. For all tests, the significance level was set at $\mathrm{P}<0.05$. 


\section{RESULTS}

\section{Clinicopathologic characteristics of patients with thyroid carcinomas}

The clinical and pathological conditions of the 126 patients are shown in Table 1. There were 11 male patients $(8.7 \%)$ and 115 female patients (91.3\%), giving a male-to-female ratio of 1:10.45. The patients' ages ranged from 19 to 73 years, with an average age of 43.1 \pm 10.6 years. There were 55 patients aged $\geq 45$ years $(43.7 \%)$ and 71 patients aged $<45$ years $(56.3 \%)$. The tumor diameters ranged from 2 to $35 \mathrm{~mm}$, with an average diameter of $10.7 \pm$ $7.3 \mathrm{~mm}$. There were multiple lesions in 6 patients $(4.8 \%)$ and single lesions in 120 patients (95.2\%). Tumor invasion of the extrathyroid tissues was observed in 7 patients (5.6\%). Twenty patients also had Hashimoto's thyroiditis (15.9\%). Central lymph node metastasis occurred in 42 patients $(33.3 \%)$. A total of 114 patients were diagnosed with stage I tumors $(90.5 \%)$, and 12 patients $(9.5 \%)$ were diagnosed with stage III tumors. BRAF gene mutations occurred in 87 patients $(69.0 \%)$.

Table 1. Clinicopathologic characteristics of 126 patients with papillary thyroid carcinomas diagnosed between October 2011 and October 2013.

\begin{tabular}{|c|c|c|}
\hline & No. of patients & $(\%)$ \\
\hline \multicolumn{3}{|l|}{ Gender } \\
\hline Male & 11 & 8.7 \\
\hline Female & 115 & 91.3 \\
\hline Age (years) & $43.1 \pm 10.6$ & \\
\hline$\geq 45$ & 55 & 43.7 \\
\hline$<45$ & 71 & 56.3 \\
\hline Tumor size (mm) & $10.7 \pm 7.3$ & \\
\hline \multicolumn{3}{|l|}{$\begin{array}{l}\text { Lesion type } \\
\text { lat }\end{array}$} \\
\hline Multiple $(\geq 2)$ & 6 & 4.8 \\
\hline Single & 120 & 95.2 \\
\hline \multicolumn{3}{|c|}{ Tumor invasion into tissues outside the thyroid gland } \\
\hline Yes & 7 & 5.6 \\
\hline No & 119 & 94.4 \\
\hline \multicolumn{3}{|c|}{ Hashimoto's thyroiditis } \\
\hline Yes & 20 & 15.9 \\
\hline No & 106 & 84.1 \\
\hline \multicolumn{3}{|c|}{ Lymph node metastasis } \\
\hline Yes & 42 & 33.3 \\
\hline No & 84 & 66.7 \\
\hline \multicolumn{3}{|l|}{ Tumor staging* } \\
\hline T1 & 114 & 90.5 \\
\hline $\mathrm{T} 3$ & 12 & 9.5 \\
\hline \multicolumn{3}{|l|}{ BRAF mutation } \\
\hline Yes & 87 & 69.0 \\
\hline No & 39 & 31.0 \\
\hline
\end{tabular}

\section{BRAF V600E mutation test results}

BRAF gene mutations were observed in 87 of the 126 patients with PTC, giving an incidence rate of $69.0 \%(87 / 126)$. The results of the experimental comparison with respect to DNA extraction and gene mutation detection in the 126 samples are shown in Figure 1. 


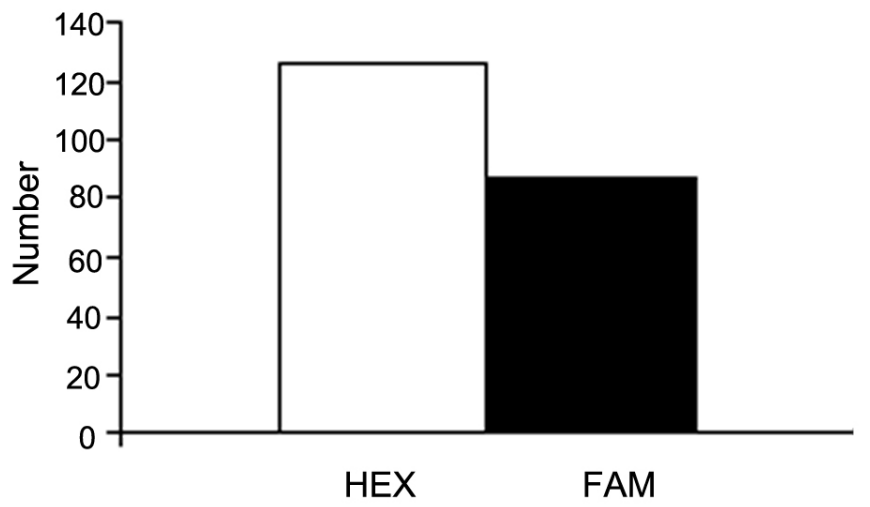

Figure 1. Comparison of the experimental results of DNA extraction and gene mutation detection in the 126 patient samples. Two types of tobacco acid pyrophosphatase (TAP) can emit fluorescence signals during reverse transcription-polymerase chain reaction. The TAP corresponding to HEX is DNA-specific, whereas the TAP corresponding to FAM is V-raf murine sarcoma viral oncogene homolog B1 (BRAF)-specific. The enhanced HEX signal in all samples suggested successful DNA extraction, and the increased FAM signal in 87 samples indicated the occurrence of BRAF mutations.

\section{Relationships between BRAF gene mutation and the clinicopathologic features of PTC}

The relationships between the BRAF gene mutation status and various clinicopathologic features of PTC are shown in Table 2. Univariate analysis uncovered that the BRAF mutation status was correlated significantly with tumor size and lymph node metastasis $(\mathrm{P}<0.05)$ but not with gender, age, the presence of multiple lesions, tumor invasion into extrathyroid tissues, Hashimoto's thyroiditis, and the tumor stage $(\mathrm{P}>0.05)$. Multivariate analysis found that only lymph node metastasis was correlated significantly with the BRAF mutation status $(\mathrm{P}<0.05)$.

Table 2. Relationships between the BRAF gene mutation status and the clinicopathologic features of PTC.

\begin{tabular}{|c|c|c|c|c|}
\hline \multirow[t]{2}{*}{ Variable (126 patients) } & Univariate analysis & \multirow[t]{2}{*}{ P value } & Multivariate analysis & \multirow[t]{2}{*}{$P$ value } \\
\hline & OR $(95 \% \mathrm{CI})$ & & OR $(95 \% \mathrm{CI})$ & \\
\hline Gender (male/female) & $2.1(0.4-10.4)$ & 0.347 & $2.6(0.5-14.6)$ & 0.265 \\
\hline Age ( $\geq 45$ years $/<45$ years $)$ & $0.6(0.3-1.4)$ & 0.249 & $1.0(0.4-2.5)$ & 0.960 \\
\hline Tumor size (I/II/III/IV) & $1.7(1.1-2.8)$ & 0.027 & $1.3(0.8-2.3)$ & 0.276 \\
\hline Multiple lesions (multiple/single) & $2.3(0.3-20.5)$ & 0.45 & $3.2(0.3-32.0)$ & 0.324 \\
\hline Tumor invasion into extrathyroid tissues (Yes/No) & $1.1(0.2-6.1)$ & 0.889 & $0.7(0.1-4.8)$ & 0.677 \\
\hline Hashimoto's thyroiditis (Yes/No) & $0.5(0.2-1.3)$ & 0.144 & $0.6(0.2-1.8)$ & 0.375 \\
\hline Central lymph node metastasis (Yes/No) & $5.0(1.8-14.1)$ & 0.002 & $4.6(1.2-16.7)$ & 0.022 \\
\hline Tumor staging (T3/T1) & $1.6(0.7-3.4)$ & 0.273 & $0.8(0.1-5.7)$ & 0.787 \\
\hline
\end{tabular}

Tumor sizes: I, $\leq 5 \mathrm{~mm}$; II, $>5$ to $10 \mathrm{~mm}$; III, $>10$ to $20 \mathrm{~mm}$; IV, $>20 \mathrm{~mm}$. Age and tumor size were used as continuous variables in univariate and multivariate analyses. BRAF: V-raf murine sarcoma viral oncogene homolog B1; OR: odds ratio; CI: confidence interval.

\section{Relationships among central lymph node metastasis, the BRAF mutation status, and tumor size}

Comparisons of the BRAF mutation statuses and characteristics of central lymph node metastasis among the patients are shown in Table 3. The difference in the average number of meta- 
static central lymph nodes between mutation-negative and mutation-positive patients was statistically significant $(\mathrm{P}<0.001)$, as was the difference in the ratio of the number of metastatic lymph nodes to the total number of removed lymph nodes $(\mathrm{P}<0.0001)$. However, the BRAF mutation status did not affect the number of surgically removed lymph nodes, and the difference between the mutation-negative and mutation-positive patients was not statistically significant $(\mathrm{P}>0.05)$.

The relationship among central lymph node metastasis, the tumor size, and the BRAF mutation status is shown in Table 4. When the tumor diameter was $\leq 10 \mathrm{~mm}$, the BRAF mutation status had no effect on lymph node metastasis $(\mathrm{P}>0.05)$. However, lymph node metastasis was not observed in the samples from mutation-negative patients with tumor diameters of $\leq 5 \mathrm{~mm}$. When the tumor diameter was $>10 \mathrm{~mm}$, the incidence of lymph node metastasis was significantly higher among BRAF mutation-positive patients than among mutation-negative patients $(\mathrm{P}<0.05)$.

Table 3. Comparison of the BRAF mutation status and the characteristics of central lymph node metastasis.

\begin{tabular}{|c|c|c|c|c|}
\hline & \multicolumn{2}{|c|}{ BRAF V600E mutation } & \multirow[t]{2}{*}{$\chi^{2 / t}$} & \multirow[t]{2}{*}{$P$ value } \\
\hline & Positive & Negative & & \\
\hline Central lymph node metastasis [patient (\%)] & & & 10.695 & 0.001 \\
\hline Yes & $37(88.1)$ & $5(11.9)$ & & \\
\hline No & $50(59.5)$ & $34(40.5)$ & & \\
\hline Number of lymph nodes removed (mean \pm standard deviation) & $4.8 \pm 4.1$ & $4.6 \pm 3.4$ & 0.217 & 0.829 \\
\hline Number of metastatic lymph nodes (mean \pm standard deviation) & $1.2 \pm 2.2$ & $0.2 \pm 0.7$ & 3.650 & $<0.001$ \\
\hline $\begin{array}{l}\text { Number of metastatic lymph nodes/number of lymph nodes } \\
\text { removed (mean } \pm \text { standard deviation) }\end{array}$ & $0.2 \pm 0.3$ & $0.06 \pm 0.2$ & 3.644 & $<0.001$ \\
\hline
\end{tabular}

BRAF: V-raf murine sarcoma viral oncogene homolog B1.

Table 4. Relationships between central lymph node metastasis, tumor size, and the BRAF mutation status.

\begin{tabular}{|c|c|c|c|c|c|c|c|}
\hline \multirow[t]{2}{*}{ Tumor size (mm) } & \multirow[t]{2}{*}{ BRAF mutation } & \multicolumn{4}{|c|}{ Central lymph node metastasis } & \multirow[t]{2}{*}{$\chi^{2}$} & \multirow[t]{2}{*}{$\mathrm{P}$ value } \\
\hline & & Yes (patients) & $\%$ & No (patients) & $\%$ & & \\
\hline \multirow[t]{3}{*}{$\leq 5^{\text {a }}$} & & & & & & 0.941 & 0.332 \\
\hline & Positive & 3 & 18.8 & 13 & 81.2 & & \\
\hline & Negative & 0 & 0 & 12 & 100 & & \\
\hline \multirow[t]{3}{*}{$>5-10^{\mathrm{b}}$} & & & & & & 1.066 & 0.302 \\
\hline & Positive & 14 & 35.9 & 25 & 64.1 & & \\
\hline & Negative & 4 & 22.2 & 14 & 77.8 & & \\
\hline \multirow[t]{3}{*}{$>10^{*}$} & & & & & & 5.510 & 0.019 \\
\hline & Positive & 20 & 62.5 & 12 & 37.5 & & \\
\hline & Negative & 1 & 11.1 & 8 & 88.9 & & \\
\hline
\end{tabular}

*In this sample, patients with tumor diameters of $>20 \mathrm{~mm}$ were all BRAF mutation-positive. Patients with tumor diameters of $>20 \mathrm{~mm}$ were all included in the $>10-\mathrm{mm}$ group, and a continuity correction was performed. ${ }^{\mathrm{a} C}$ Continuity correction; ${ }^{\mathrm{b}}$ Pearson $\chi^{2}$ test. BRAF: V-raf murine sarcoma viral oncogene homolog B1.

\section{DISCUSSION}

To date, studies have identified more than 40 different BRAF gene mutations in human malignancies (Davies et al., 2002). The T1799A point mutation is the most common mutation, accounting for $90 \%$ of all BRAF gene mutations (Garnett and Marais, 2004). Research illustrated that BRAF gene mutations, which can lead to tumor cell proliferation and differentiation (Kondo et al., 2006), represent the most common genetic event in thyroid carcinomas; the incidence of this event is the highest in patients with PTC, occurring in $29-88 \%$ of all patients (Kim et al., 2004; Mathur et al., 2011; Chakraborty et al., 2012). Meanwhile, PTC is 
the most common pathological type of thyroid cancer, accounting for approximately $80-90 \%$ of all cases (Moo et al., 2010; Byrd et al., 2012). Therefore, a study of the relationship between the BRAF mutation status and PTC-associated clinicopathologic factors could help to identify the occurrence of thyroid carcinoma cells and the mechanisms of PTC development and also help clinicians to provide better treatment for this disease and better evaluations of patient prognosis. However, the link between BRAF mutations and certain clinicopathologic features of PTC remains controversial. Therefore, this study aimed to investigate the correlations of the BRAF gene mutation status with the clinicopathologic features of PTC and central lymph node metastasis.

This study found a BRAF mutation incidence rate of $69.0 \%$, which is higher than the recently reported average rate of $62.5 \%$ (Brahma et al., 2013). Recently, Mathur et al. (2011) found that the BRAF mutation incidence was as high as $88 \%$ in a 15 -year study sample and also noted that the high prevalence of BRAF gene mutations could increase the incidence of thyroid carcinoma. Xing et al. (2005) found that BRAF mutations were closely associated with poor clinicopathologic results in patients with thyroid carcinoma and suggested that BRAF mutations could silence tumor suppressor genes via methylation, therefore resulting in a reduced ability of PTC lesions to take up iodine, a higher degree of PTC malignancy, and an eventual increase in the PTC mortality rate. Patient follow-up data were not available in the current study. Therefore, the best use of BRAF V600E gene status information for controlling the risk of death in patients with PTC remains to be further studied.

The traditional risk factors for malignant thyroid carcinoma include advanced age, male gender, a larger tumor diameter, tumor invasion into extrathyroid tissues, local lymph node metastasis, distant metastasis, and an advanced pathologic stage. Several studies have proven that BRAF mutations can significantly increase the incidence of tumor invasion into extrathyroid tissues, lymph node metastasis, and higher TNM staging (Xing et al., 2005; Lupi et al., 2007; Kim et al., 2012). In this study, no correlations were found between the BRAF mutation status and gender, age, the presence of multiple lesions, tumor invasion into extrathyroid tissues, Hashimoto's thyroiditis, and tumor stage. This finding might be related to the sample size, statistical standards, and different standards for tumor clinicopathologic classification used in this study, all of which should be explored in further studies. In this study, we found that BRAF gene mutation positivity correlated significantly with tumor size and lymph node metastasis $(\mathrm{P}<0.05)$, a finding that was consistent with the results of a study by Frasca et al. (2008). The results proved that BRAF gene mutations were closely related to invasive tumor behavior. In a multivariate analysis, BRAF gene mutation positivity correlated only with lymph node metastasis $(\mathrm{P}<0.05)$; therefore, BRAF gene mutation positivity could be considered an independent predictor of the risk of central lymph node metastasis. This finding was consistent with the results reported by Lupi et al. (2007), who studied a bulk sample of up to 500 patients. However, Lupi et al. believed that an advanced tumor stage and PTC invasion into the extrathyroid tissues were also independent predictors of central lymph node metastasis. The results of the present study suggested that the importance of central lymph node dissection should be emphasized in patients with PTC who have been preoperatively diagnosed with BRAF mutation positivity and that regional lymphatic and adipose tissue dissection should be routinely conducted. However, a study by Chakraborty et al. (2012) demonstrated that BRAF mutations could not be an independent predictor of the risk of lymph node metastasis in patients with PTC. This is therefore another focus of debate. We believe that increased numbers of study samples, additional new data, improved follow-up visits, and a better understanding of tumor staging will clarify this argument. 
The present study further analyzed the relationship among central lymph node metastasis, the BRAF mutation status, and tumor size and found that BRAF mutation significantly increased the incidence of central lymph node metastasis in patients with PTC $(\mathrm{P}<0.05)$. This finding is consistent with the conclusion reached by Kim et al. (2012). Although BRAF mutation positivity did not increase the total number of lymph nodes removed, it significantly increased the ratio of metastatic lymph nodes to the total number removed, thus demonstrating the influences and predictive effects of BRAF mutations on central lymph node metastasis in patients with PTC. In addition, another study found that the importance of central lymph node dissection increased as the tumor diameters increased in BRAF mutation-positive patients. When the tumor diameters was $>10 \mathrm{~mm}$, the incidence of lymph node metastasis was significantly higher in BRAF mutation-positive patients than in BRAF mutation-negative patients $(\mathrm{P}<0.05)$. However, when the tumor diameter was $\leq 10 \mathrm{~mm}$, BRAF mutation positivity had no effect on lymph node metastasis $(\mathrm{P}>0.05)$. However, in this study, lymph node metastasis was not detected in the samples from mutation-negative patients with tumor diameters of $\leq 5$ $\mathrm{mm}$. To date, studies of how BRAF gene mutations affect the selection of the surgical approach used to treat PTC have rarely been reported. Although the use of central lymph node dissection as a routine surgical approach for the treatment of PTC has been generally accepted in the academic field, central lymph node dissection surgery can lead to increased surgical injury-related adverse effects in patients; therefore, a study to determine the types of patients suitable for central lymph node dissection remains a focus of academic research. The current study speculates that for mutation-negative patients with tumor diameters of $\leq 5 \mathrm{~mm}$, the need for central lymph node dissection should be re-examined. Given the limitation of the study sample size, this conclusion should be drawn from additional studies with larger sample sizes.

In summary, this study found a BRAF V600E mutation incidence rate of $69.0 \%$ among patients with PTC and that BRAF mutation positivity correlated with highly invasive biological tumor behaviors and served as an independent predictor of the risk of central lymph node metastasis of PTC. Regarding preoperatively detected BRAF mutation-positive PTC, more attention should be paid to the importance of central lymph node dissection in patients with large tumor diameters, and regional lymphatic and adipose tissue dissection should be routinely conducted. However, the need for central lymph node dissection should be re-examined in mutation-negative patients with tumor diameters of $\leq 5 \mathrm{~mm}$.

\section{REFERENCES}

Brahma B, Yulian ED, Ramli M, Setianingsih I, et al. (2013). Surgical perspective of T1799A BRAF mutation diagnostic value in papillary thyroid carcinoma. Asian Pac. J. Cancer Prev. 14: 31-37.

Byrd JK, Yawn RJ, Wilhoit CS, Sora ND, et al. (2012). Well differentiated thyroid carcinoma: current treatment. Curr. Treat. Options Oncol. 13: 47-57.

Chakraborty A, Narkar A, Mukhopadhyaya R, Kane S, et al. (2012). BRAF V600E mutation in papillary thyroid carcinoma: significant association with node metastases and extra thyroidal invasion. Endocr. Pathol. 23: 83-93.

Davies H, Bignell GR, Cox C, Stephens P, et al. (2002). Mutations of the BRAF gene in human cancer. Nature 417: 949-954.

Frasca F, Nucera C, Pellegriti G, Gangemi P, et al. (2008). BRAF(V600E) mutation and the biology of papillary thyroid cancer. Endocr. Relat. Cancer 15: 191-205.

Garnett MJ and Marais R (2004). Guilty as charged: B-RAF is a human oncogene. Cancer Cell 6: 313-319.

Kim KH, Kang DW, Kim SH, Seong IO, et al. (2004). Mutations of the BRAF gene in papillary thyroid carcinoma in a Korean population. Yonsei Med. J. 45: 818-821.

Kim SJ, Lee KE, Myong JP, Park JH, et al. (2012). BRAF V600E mutation is associated with tumor aggressiveness in papillary thyroid cancer. World J. Surg. 36: 310-317. 
Kondo T, Ezzat S and Asa SL (2006). Pathogenetic mechanisms in thyroid follicular-cell neoplasia. Nat. Rev. Cancer 6: 292-306.

Lupi C, Giannini R, Ugolini C, Proietti A, et al. (2007). Association of BRAF V600E mutation with poor clinicopathological outcomes in 500 consecutive cases of papillary thyroid carcinoma. J. Clin. Endocrinol. Metab. 92: 4085-4090.

Mathur A, Moses W, Rahbari R, Khanafshar E, et al. (2011). Higher rate of BRAF mutation in papillary thyroid cancer over time: a single-institution study. Cancer 117: 4390-4395.

Montero PH, Ibrahimpasic T, Nixon IJ and Shaha AR (2014). Thyroid metastasectomy. J. Surg. Oncol. 109: 36-41.

Moo TA, McGill J, Allendorf J, Lee J, et al. (2010). Impact of prophylactic central neck lymph node dissection on early recurrence in papillary thyroid carcinoma. World J. Surg. 34: 1187-1191.

Sadowski BM, Snyder SK and Lairmore TC (2009). Routine bilateral central lymph node clearance for papillary thyroid cancer. Surgery 146: 696-703; discussion 703-695.

Stratmann M, Sekulla C, Dralle H and Brauckhoff M (2012). Current TNM system of the UICC/AJCC: the prognostic significance for differentiated thyroid carcinoma. Chirurg 83: 646-651.

Xing M, Westra WH, Tufano RP, Cohen Y, et al. (2005). BRAF mutation predicts a poorer clinical prognosis for papillary thyroid cancer. J. Clin. Endocrinol. Metab. 90: 6373-6379. 